JOURNAL OF

SYMPLECTIC GEOMETRY

Volume 11, Number 1, 93-108, 2013

\title{
NONCOMMUTATIVE POISSON BRACKETS ON LODAY ALGEBRAS AND RELATED DEFORMATION QUANTIZATION
}

\author{
KYOUSUKE UCHINO
}

\begin{abstract}
Given a Lie algebra, there uniquely exists a Poisson algebra that is called a Lie-Poisson algebra over the Lie algebra. We will prove that given a Loday/Leibniz algebra there exists uniquely a noncommutative Poisson algebra over the Loday algebra. The noncommutative Poisson algebras are called the Loday-Poisson algebras. In the super/graded cases, the Loday-Poisson bracket is regarded as a noncommutative version of classical (linear) Schouten-Nijenhuis bracket. It will be shown that the Loday-Poisson algebras form a special subclass of Aguiar's dual-prePoisson algebras. We also study a problem of deformation quantization over the Loday-Poisson algebra. It will be shown that the polynomial Loday-Poisson algebra is deformation quantizable and that the associated quantum algebra is Loday's associative dialgebra.
\end{abstract}

\section{Introduction}

A Loday algebra (also called Leibniz algebra $[\mathbf{8}, \mathbf{1 0}]$ ) is an algebra equipped with a binary bracket satisfying the Leibniz identity,

$$
\left[x_{1},\left[x_{2}, x_{3}\right]\right]=\left[\left[x_{1}, x_{2}\right], x_{3}\right]+\left[x_{2},\left[x_{1}, x_{3}\right]\right] .
$$

This new type algebra was introduced by Loday. Hence, it is called a Loday algebra. A Lie algebra is clearly a Loday algebra whose bracket is anti-commutative. However, the Loday bracket is not anti-commutative in general.

The Loday algebras arise in several area of mathematics, for example, as Loday algebroids in Nambu-mechanics [6]; as Courant algebroids in Poisson geometry; in representation theory of Lie algebras and so on. 
Since Loday algebras are considered to be noncommutative analogues of Lie algebras, it is natural to ask what type of algebra is noncommutative analogue of Lie-Poisson algebra. The aim of this note is to solve this question.

In first, we recall classical Lie-Poisson algebras. If $\mathfrak{g}$ is a Lie algebra, then the polynomial functions $S(\mathfrak{g})$ becomes a Poisson algebra, that is, the Lie-Poisson algebra. The Lie-Poisson bracket is characterized by the following 4-conditions; (C1) The Lie-Poisson algebras are Lie- and Commutativealgebras. (C2) The Lie-Poisson bracket is a biderivation with respect to the associative multiplication on $S(\mathfrak{g})$. (C3) The base algebra $\mathfrak{g}$ is a Lie subalgebra of $S(\mathfrak{g})$. (C4) The Lie-Poisson bracket on $S(\mathfrak{g})$ is free over the Lie bracket on $\mathfrak{g}$.

Let us consider noncommutative analogues of the conditions $(\mathrm{C} 1)-(\mathrm{C} 4)$. We assume that $\mathfrak{g}$ is a Loday algebra and that $\mathcal{A}$ is a certain algebra generated from $\mathfrak{g}$. The noncommutative analogues of $(\mathrm{C} 1)-(\mathrm{C} 4)$ are respectively as follows:

(A1) The algebra $\mathcal{A}$ is a Loday- and associative-algebra, not necessarily commutative.

(A2) The Loday bracket on $\mathcal{A}$ is a biderivation with respect to the associative multiplication.

(A3) The base algebra $\mathfrak{g}$ is a Loday subalgebra of $\mathcal{A}$.

(A4) The Loday bracket on $\mathcal{A}$ is free over the bracket on $\mathfrak{g}$.

We notice that Aguiar's dual-prePoisson algebras [1] satisfy (A1)-(A3). We will prove that given a Loday algebra there exists a dual-prePoisson algebra satisfying also (A4) (Theorem 4.4 below). We call this special dualprePoisson algebra a Loday-Poisson algebra, which is considered to be a noncommutative version of Lie-Poisson algebra. As an application, we will study super Loday-Poisson brackets, which are considered as noncommutative analogues of classical (linear) Schouten-Nijenhuis (SN) brackets (see Section 4.2 below).

To understand the dual-prePoisson algebras we will study a category of linear mappings $\mathcal{L} \mathcal{M}$ in $[\mathbf{9}]$. It is known that Loday algebras are Lie gebras in $\mathcal{L} \mathcal{M}$ (so-called Lie objects). We will introduce the notion of Poisson gebra in $\mathcal{L} \mathcal{M}$, which are called Poisson objects (see Section 3 below). It will be shown that the Loday-Poisson algebra is the free Poisson gebra defined over a Lie gebra.

We also study an algebraic quantization of polynomial Loday-Poisson algebras. It will be shown that the polynomial Loday-Poisson algebras are deformation quantizable in the sense of Kontsevich [7] and that the quantum algebras are associative dialgebras in [10]. Namely, given a LodayPoisson algebra of polynomials, there exists a unique associative dialgebra parameterized by $\hbar$ whose classical limit is the Loday-Poisson algebra. It is 
a difficult problem whether Nambu-Poisson brackets are quantizable. Since the Nambu-Poisson brackets are equivalently Loday brackets, our results provide a hope that Nambu-Poisson brackets are quantizable.

The paper is organized as follows. In Section 2, we recall three noncommutative algebras, i.e., Loday algebras, perm-algebras of Chapoton $[\mathbf{2}, \mathbf{3}]$ and dual-prePoisson algebras. In Section 3, we study a category of linear mappings. In Section 4, we give the main theorem of this note, namely, the problem above is solved. In Section 5, we discuss the deformation quantization for the Loday-Poisson algebras.

\section{New type algebras}

2.1. Loday/Leibniz algebras $[\mathbf{8}, \mathbf{1 0}]$. A Loday algebra $(\mathfrak{g},[]$,$) is, by defi-$ nition, a vector space equipped with a binary bracket product which satisfies the Leibniz identity,

$$
[x,[y, z]]=[[x, y], z]+[y,[x, z]],
$$

where $x, y, z \in \mathfrak{g}$. If the bracket is anti-commutative, then the Loday algebra is a Lie algebra. Thus, Loday algebras are considered to be noncommutative analogues of Lie algebras.

We consider a subspace of the Loday algebra composed of the symmetric brackets,

$$
\mathfrak{g}^{a n n}:=\{[x, y]+[y, x] \mid x, y \in \mathfrak{g}\} .
$$

This becomes a two side ideal of the Loday algebra because

$$
\begin{aligned}
& {[[x, x], y]=[x,[x, y]]-[x,[x, y]]=0,} \\
& {[x,[y, y]]=[[x, y], y]+[y,[x, y]],}
\end{aligned}
$$

for any $x, y \in \mathfrak{g}$. Hence, the quotient space $\mathfrak{g}_{\text {Lie }}:=\mathfrak{g} / \mathfrak{g}^{\text {ann }}$ becomes a Lie algebra. The projection $\mathfrak{g} \rightarrow \mathfrak{g}_{\text {Lie }}$, which is called a Liezation, is a universal arrow, that is,

$$
\operatorname{Hom}_{\text {Lod }}(\mathfrak{g}, \square \mathfrak{h}) \cong \operatorname{Hom}_{\text {Lie }}\left(\mathfrak{g}_{\text {Lie }}, \mathfrak{h}\right),
$$

where $\mathfrak{h}$ is an arbitrary Lie algebra and $\square$ is the forgetful functor from the category of Lie algebras to the one of Loday algebras. One can define a Lie algebra action $\mathfrak{g}_{\text {Lie }} \otimes \mathfrak{g} \rightarrow \mathfrak{g}$ by

$$
\operatorname{ad}(\bar{x})(y):=[x, y] .
$$

The Loday bracket is identified with this action.

The free Loday algebra over a vector space has the following form:

$$
\mathcal{F}_{\mathcal{L}_{\text {od }}}(V)=\bar{T} V:=\bigoplus_{n \geq 1} V^{\otimes n}
$$


Here, the Loday bracket is defined by

$$
\left[x_{1},\left[x_{2},\left[\ldots,\left[x_{n-1}, x_{n}\right]\right]\right]\right]:=x_{1} \otimes \cdots \otimes x_{n},
$$

for any $x, \in V$.

2.2. Perm-algebras $[2,3]$. An associative algebra $(A, *)$ is called a permutation algebra, or perm-algebra for short, if $A$ satisfies

$$
(x * y) * z=(y * x) * z,
$$

where $x, y, z \in A$.

We consider a monomial on the perm-algebra. $x_{1} * \cdots x_{n-1} * x_{n} \in A$. Up to the $(-) * x_{n}, x_{1} * \cdots * x_{n-1}$ is regarded as a monomial of commutative algebra. This implies that the free perm-algebra over a vector space $V$ has the following form:

$$
\mathcal{F}_{\mathcal{P}_{\text {erm }}}(V)=S(V) \otimes V,
$$

where $S(V)$ is the free "unital" commutative algebra over $V$, or the polynomial algebra over $V$. The perm-multiplication on $\mathcal{F}_{\mathcal{P e r m}}(V)$ is defined by

$$
(f \otimes x) *(g \otimes y):=f x g \otimes y,
$$

where $f, g \in S(V)$ and $x, y \in V$.

Example 2.1. Let $(C, d)$ be a differential graded $(\mathrm{dg})$ commutative algebra. Define a new product on $C$ by

$$
x * y:=-(-1)^{|x|}(d x) y .
$$

Then $(C, *)$ becomes a graded perm-algebra.

2.3. Dual-prePoisson/Aguiar algebras [1]. Let $(A, *,\{\}$,$) be a perm-$ algebra equipped with a Loday bracket. It is called a dual-prePoisson algebra $^{1}$, if the following three conditions are satisfied:

$$
\begin{aligned}
& \{x, y * z\}=\{x, y\} * z+y *\{x, z\}, \\
& \{x * y, z\}=x *\{y, z\}+y *\{x, z\}, \\
& \{x, y\} * z=-\{y, x\} * z,
\end{aligned}
$$

where $x, y, z \in A$. We call the axioms (2.4) and (2.5) the biderivation conditions of dual-prePoisson algebra. In the graded cases, (2.4)-(2.6) have the following form,

$$
\begin{aligned}
& \{x, y * z\}=\{x, y\} * z+(-1)^{(|x|-n)|y|} y *\{x, z\}, \\
& \{x * y, z\}=x *\{y, z\}+(-1)^{|x||y|} y *\{x, z\}, \\
& \{x, y\} * z=-(-1)^{(|x|-n)(|y|-n)}\{y, x\} * z,
\end{aligned}
$$

\footnotetext{
${ }^{1}$ The operad of dual-prePoisson algebras is the Koszul "dual" of the one of pre-Poisson algebras. As such the name is.
} 
where $|x|,|y|$ are the degrees of elements and $n$ is the one of the bracket. The perm-multiplication also satisfies the natural sign convention,

$$
x * y * z=(-1)^{|x||y|} y * x * z,
$$

where we put $|*|:=0$.

We sometimes call the dual-prePoisson algebra an Aguiar algebra, because the name "dual-prePoisson" is long.

We consider a subspace of the dual-prePoisson algebra generated by the symmetric elements,

$$
A^{\text {ann }}:=\langle x * y-y * x, \quad\{x, y\}+\{y, x\}\rangle .
$$

This is a two-side ideal of the dual-prePoisson algebra. Hence, the quotient space $A / A^{\text {ann }}$ becomes a Poisson algebra. One can easily check that $A \rightarrow$ $A / A^{\text {ann }}$ is the universal arrow, like the Liezation $\mathfrak{g} \rightarrow \mathfrak{g}_{\text {Lie }}$. So, we call the projection $A \rightarrow A / A^{\text {ann }}$ a Poissonization of dual-prePoisson algebra.

From the axioms of dual-prePoisson algebras, we obtain $A^{\text {ann }} * A=0$ and $\left\{A^{\text {ann }}, A\right\}=0$. Under a mild assumption, $A$ is considered to be a semi-direct product algebra

$$
A=A_{\text {Poiss }} \ltimes A^{\text {ann }},
$$

where $A_{\text {Poiss }}$ is the result of the Poissonization $A \rightarrow A_{\text {Poiss }}$. Conversely, given a Poisson algebra $P$ and a left Poisson module $M$, the semi-direct product $P \ltimes M$ becomes a dual-prePoisson algebra, whose dual-prePoisson products are defined by

$$
\begin{aligned}
\left(p_{1}+m_{1}\right) *\left(p_{2}+m_{2}\right) & :=p_{1} p_{2}+p_{1} \cdot m_{2}, \\
\left\{p_{1}+m_{1}, p_{2}+m_{2}\right\} & :=\left\{p_{1}, p_{2}\right\}+\left\{p_{1}, m_{2}\right\},
\end{aligned}
$$

where $p_{1}, p_{2} \in P, m_{1}, m_{2} \in M$ and where $\left\{p_{1}, p_{2}\right\}$ is the Poisson bracket on $P$ and $p_{1} \cdot m_{2},\left\{p_{1}, m_{2}\right\}$ are the left-module structures.

Example 2.2. Let $\left(P, d,\{,\}^{\prime}\right)$ be a dg Poisson algebra with a Poisson bracket $\{,\}^{\prime}$. Define the new products by

$$
\begin{gathered}
x * y:=-(-1)^{|x|}(d x) y, \\
\{x, y\}:=-(-1)^{|x|}\{d x, y\}^{\prime} .
\end{gathered}
$$

Then $(P, *,\{\}$,$) becomes a graded dual-prePoisson algebra.$

We consider the free dual-prePoisson algebra. By the biderivation properties (2.4) and (2.5), a monomial of dual-prePoisson algebra, $m$, is decomposed into the form,

$$
m=\sum_{m^{\prime}, n} m^{\prime} * n
$$


where $m^{\prime}$ and $n$ are monomials and where $n$ has no *. For example, $\{\{x, y * z\}, w\}=\{x, y\} *\{z, w\}+z *\{\{x, y\}, w\}+y *\{\{x, z\}, w\}+\{x, z\} *\{y, w\}$, where $\{z, w\},\{\{x, y\}, w\},\{\{x, z\}, w\}$ and $\{y, w\}$ are $n$ in (2.9).

Since a dual-prePoisson algebra is a Loday algebra, $n$ is regarded as a monomial of Loday algebra. From the axioms of dual-prePoisson algebra, $(x * y) * z=(y * x) * z$ and $\{x, y\} * z=-\{y, x\} * z, m^{\prime}$ is regarded as a monomial of Poisson algebra up to $(-) * A$. From this observation, we obtain

Proposition 2.3. Let $V$ be a vector space. The free dual-prePoisson algebra over $V$ has the following form:

$$
\mathcal{F}_{\mathcal{P}_{\text {oiss }}}(V) \otimes \mathcal{F}_{\mathcal{L}_{\text {od }}}(V)
$$

where $\mathcal{F}_{\mathcal{P}_{\text {oiss }}}(V)$ is the free "unital" Poisson algebra.

Proof. We will give a proof of the proposition in Section 4.

Proposition 2.4. $\overline{\mathcal{F}}_{\mathcal{P}_{\text {oiss }}}(V) \cong\left(\mathcal{F}_{\mathcal{P}_{\text {oiss }}}(V) \otimes \mathcal{F}_{\mathcal{L} \text { od }}(V)\right)_{\text {Poiss }}$, where $\overline{\mathcal{F}}_{\mathcal{P}_{\text {oiss }}(V)}$ is the free Poisson algebra of nonunital.

Proof. Because the Poissonization is universal.

\section{Category of linear mappings}

The category of linear maps $[\mathbf{9}]$, which is denoted by $\mathcal{L} \mathcal{M}$, is a category whose objects are linear maps $\rho: V_{1} \rightarrow V_{0}$ and morphisms $\left(F_{1}, F_{0}\right)$ are commutative diagrams of liner maps:

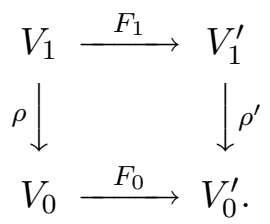

One can define a tensor product on $\mathcal{L} \mathcal{M}$ by

$$
\begin{aligned}
& \rho \otimes_{\mathcal{L M}} \rho^{\prime}:=\rho \otimes 1+1 \otimes \rho^{\prime}, \\
& \rho \otimes_{\mathcal{L} \mathcal{M}} \rho^{\prime}:\left(V_{1} \otimes V_{0}^{\prime}\right) \oplus\left(V_{0} \otimes V_{1}^{\prime}\right) \rightarrow V_{0} \otimes V_{0}^{\prime} .
\end{aligned}
$$

If $F:=\left(F_{1}, F_{0}\right)$ and $G:=\left(G_{1}, G_{0}\right)$ are morphisms in $\mathcal{L} \mathcal{M}$, then the tensor product of $F$ and $G$ has the following form:

$$
F \otimes_{\mathcal{L M}} G=\left(F_{1} \otimes G_{0} \oplus F_{0} \otimes G_{1}, F_{0} \otimes G_{0}\right) .
$$

It is known that $\left(\mathcal{L} \mathcal{M}, \otimes_{\mathcal{L M}}\right)$ becomes a symmetric monoidal category.

A Lie algebra object (shortly, Lie object) is by definition a Lie (al)gebra in $\mathcal{L} \mathcal{M}$. That is, a Lie object is a linear mapping $\rho: \mathfrak{g}_{1} \rightarrow \mathfrak{g}_{0}$ equipped with 
a bracket morphism $\mu=\left(\mu_{1}, \mu_{0}\right)$,

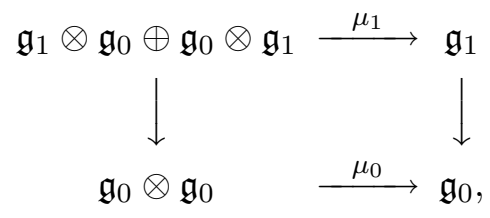

satisfying the axioms of Lie algebras, i.e., skewsymmetry and Jacobi law,

$$
\begin{aligned}
& \mu(21)=-\mu, \\
& \mu\left(1 \otimes_{\mathcal{L M}} \mu\right)=\mu\left(\mu \otimes_{\mathcal{L M}} 1\right)+\mu\left(1 \otimes_{\mathcal{L M}} \mu\right)(213),
\end{aligned}
$$

where (21), (213) are usual actions of symmetric groups $S_{2}, S_{3}$, respectively. The morphisms between Lie objects are defined by the usual manner.

If $\rho: \mathfrak{g}_{1} \rightarrow \mathfrak{g}_{0}$ is a Lie object equipped with a bracket $\left(\mu_{1}, \mu_{0}\right)$, then $\left(\mathfrak{g}_{0}, \mu_{0}\right)$ becomes a Lie algebra and $\left(\mathfrak{g}_{1}, \mu_{1}\right)$ becomes a $\mathfrak{g}_{0}$-bimodule satisfying the equivariant condition,

$$
\rho[\rho(x), y]_{\mu_{1}}=[\rho(x), \rho(y)]_{\mu_{0}},
$$

for any $x, y \in \mathfrak{g}_{1}$. The natural bracket, $[x, y]_{\text {Lod }}:=[\rho(x), y]_{\mu_{1}}$, becomes a Loday bracket on $\mathfrak{g}_{1}$.

Proposition 3.1 ([9]). The Liezation $\mathfrak{g} \rightarrow \mathfrak{g}_{\text {Lie }}$ is a Lie object and an arrow $\mathfrak{g} \Rightarrow\left(\mathfrak{g} \rightarrow \mathfrak{g}_{\text {Lie }}\right)$ is an adjoint functor with respect to the forgetful functor, $\left(\mathfrak{h}_{1} \rightarrow \mathfrak{h}_{0}\right) \Rightarrow h_{1}$, i.e.,

$$
\operatorname{Hom}_{\text {Lieobj }}\left(\mathfrak{g} \rightarrow \mathfrak{g}_{\text {Lie }}, \mathfrak{h}_{1} \rightarrow \mathfrak{h}_{0}\right) \cong \operatorname{Hom}_{\text {Lod }}\left(\mathfrak{g}, \mathfrak{h}_{1}\right),
$$

where LHS is the space of morphisms between Lie objects.

Proof. (Sketch) Let $\mathfrak{g}$ be a Loday algebra. The Liezation $\mathfrak{g} \rightarrow \mathfrak{g}_{\text {Lie }}$ has a bracket

$$
\mu=\left(\begin{array}{l}
\mu_{1} \\
\mu_{0}
\end{array}\right),
$$

where $\mu_{0}$ is the Lie bracket on $\mathfrak{g}_{\text {Lie }}$ and $\mu_{1}$ is the action of $\mathfrak{g}_{\text {Lie }}$ to $\mathfrak{g}$. From (3.3),

$$
\mu(1 \otimes \mathcal{L M} \mu)=\mu\left(\begin{array}{c}
1 \otimes \mu_{0} \oplus 1 \otimes \mu_{1} \\
1 \otimes \mu_{0}
\end{array}\right)=\left(\begin{array}{c}
\mu_{1}\left(1 \otimes \mu_{0} \oplus 1 \otimes \mu_{1}\right) \\
\mu_{0}\left(1 \otimes \mu_{0}\right)
\end{array}\right) .
$$

By a direct computation, one can check the Jacobi identity.

Given a Loday algebra homomorphism $\mathfrak{g} \rightarrow \mathfrak{h}_{1}$ in $\operatorname{Hom}_{\text {Lod }}\left(\mathfrak{g}, \mathfrak{h}_{1}\right)$, by the universality of the Liezation $\mathfrak{g} \rightarrow \mathfrak{g}_{\text {Lie }}$, there exists a unique Lie algebra morphism of $\mathfrak{g}_{\text {Lie }} \rightarrow \mathfrak{h}_{0}$. Thus we obtain a morphism of Lie objects.

We introduce the notion of Poisson object. The Poisson objects are by definition linear mappings $P_{1} \rightarrow P_{0}$ equipped with the pairs of commutative 
associative multiplications $\nu$ and Lie brackets $\mu$ satisfying the distributive law,

$$
\mu\left(\nu \otimes_{\mathcal{L M}} 1\right)=\nu\left(1 \otimes_{\mathcal{L M}} \mu\right)+\nu\left(1 \otimes_{\mathcal{L M}} \mu\right)(213) .
$$

The morphisms between Poisson objects are also defined by the usual manner.

If $P_{1} \rightarrow P_{0}$ is a Poisson object, then $P_{0}$ becomes a Poisson algebra and $P_{1}$ becomes a $P_{0}$-bimodule satisfying certain equivariant conditions. Then, $P_{1}$ becomes a dual-prePoisson algebra by a similar method with (2.7) and (2.8). The Poisson version of Proposition 3.1 is as follows.

Proposition 3.2. If $A$ is a dual-prePoisson algebra, the Poissonization $A \rightarrow$ $A_{\text {Poiss }}$ is a Poisson object in $\mathcal{L} \mathcal{M}$, and in a similar way the arrow $A \Rightarrow(A \rightarrow$ $\left.A_{\text {Poiss }}\right)$ has the universality

$$
\operatorname{Hom}_{\text {Poissobj }}\left(A \rightarrow A_{\text {Poiss }}, P_{1} \rightarrow P_{0}\right) \cong \operatorname{Hom}_{\text {Agu }}\left(A, P_{1}\right),
$$

where LHS (resp. RHS) is the space of morphisms between Poisson objects (resp. Aguiar/dual-prePoisson algebras) and where $P_{1} \rightarrow P_{0}$ is an arbitrary Poisson object.

\section{Loday-Poisson algebras}

In this section we solve the question in Introduction.

4.1. Polynomial cases. Let $\mathfrak{g}$ be a Loday algebra. We define a space of nonstandard polynomial functions on the dual space $\mathfrak{g}^{*}$ as

$$
\mathcal{A}^{\text {poly }}\left(\mathfrak{g}^{*}\right):=S\left(\mathfrak{g}_{\text {Lie }}\right) \otimes \mathfrak{g} .
$$

Here $1 \otimes \mathfrak{g}(\cong \mathfrak{g})$ is the space of linear functions on $\mathfrak{g}^{*}$. An associative multiplication on $\mathcal{A}^{\text {poly }}\left(\mathfrak{g}^{*}\right)$ is defined by

$$
(f \otimes x) *(g \otimes y)=f \bar{x} g \otimes y,
$$

where $f, g \in S\left(\mathfrak{g}_{\text {Lie }}\right), x, y \in \mathfrak{g}$ and $\bar{x}$ is the image of $x$ by the Liezation $\mathfrak{g} \rightarrow \mathfrak{g}_{\text {Lie }}$.

Lemma 4.1. The space $\left(\mathcal{A}^{\text {poly }}\left(\mathfrak{g}^{*}\right), *\right)$ becomes a perm-algebra.

We define an action of $S\left(\mathfrak{g}_{\text {Lie }}\right)$ to $\mathcal{A}^{\text {poly }}\left(\mathfrak{g}^{*}\right)$. For any $f \in S\left(\mathfrak{g}_{\text {Lie }}\right), g \otimes y \in$ $\mathcal{A}^{\text {poly }}\left(\mathfrak{g}^{*}\right)$,

$$
\begin{aligned}
\{f, g \otimes y\} & :=\{f, g\} \otimes y+g\{f, y\}, \\
\left\{\bar{x}_{1} \cdots \bar{x}_{n}, y\right\} & :=\sum_{i} \bar{x}_{1} \cdots \bar{x}_{i-1} \bar{x}_{i+1} \cdots \bar{x}_{n} \otimes\left[x_{i}, y\right],
\end{aligned}
$$

where $\{f, g\}$ is the Poisson brackets on $S\left(\mathfrak{g}_{\text {Lie }}\right), \bar{x}_{1} \cdots \bar{x}_{n}$ is a monomial in $S\left(\mathfrak{g}_{\text {Lie }}\right)$ and the bracket $\{f, y\}$ in (4.1) is defined by (4.2) which is well-defined (cf. (2.1)). For example, $\{\bar{x} \bar{y}, g \otimes z\}=\{\bar{x} \bar{y}, g\} \otimes z+g \bar{x} \otimes[y, z]+g \bar{y} \otimes[x, z]$. 
Definition 4.2. For any $f \otimes x, g \otimes y \in \mathcal{A}^{\text {poly }}\left(\mathfrak{g}^{*}\right)$,

$$
\{f \otimes x, g \otimes y\}:=\{f \bar{x}, g \otimes y\} .
$$

We remark that the bracket (4.3) is not skewsymmetric, even if $\mathfrak{g}$ is Lie.

Lemma 4.3. Define a projection $\mathcal{A}^{\text {poly }}\left(\mathfrak{g}^{*}\right) \rightarrow \bar{S}\left(\mathfrak{g}_{\text {Lie }}\right)$ by

$$
\overline{f \otimes x}=f \bar{x},
$$

where $\bar{S}\left(\mathfrak{g}_{\text {Lie }}\right)$ is the Lie-Poisson algebra of nonunital. The projection preserves the perm-multiplication and the bracket on $\mathcal{A}^{\text {poly }}\left(\mathfrak{g}^{*}\right)$,

$$
\begin{aligned}
\overline{(f \otimes x) *(g \otimes y)} & =(f \bar{x})(g \bar{y}), \\
\overline{\{f \otimes x, g \otimes y\}} & =\{f \bar{x}, g \bar{y}\} .
\end{aligned}
$$

The main result of this note is as follows.

Theorem 4.4. The algebra $\left(\mathcal{A}^{\text {poly }}\left(\mathfrak{g}^{*}\right), *,\{\},\right)$ is the free dual-prePoisson algebra over $\mathfrak{g}$ and $\mathfrak{g}$ is a subalgebra of $\mathcal{A}^{\text {poly }}\left(\mathfrak{g}^{*}\right)$. Namely $\mathcal{A}^{\text {poly }}\left(\mathfrak{g}^{*}\right)$ satisfies the conditions (A1)-(A4) in the Introduction section.

Proof. We show that the bracket (4.3) satisfies the Leibniz identity. It suffices to prove that the action (4.1) is Lie algebraic.

Lemma 4.5. For any $f, g \in S\left(\mathfrak{g}_{\text {Lie }}\right), h \otimes z \in \mathcal{A}^{\text {poly }}\left(\mathfrak{g}^{*}\right), L(f, g, h \otimes z)=0$, where $L$ is the Leibnizator $L(1,2,3):=\{1,\{2,3\}\}-\{\{1,2\}, 3\}-\{2,\{1,3\}\}$.

Proof. By the biderivation property, we have

$$
L(f, g, h \otimes z)=L(f, g, h) \otimes z+h L(f, g, z)=h L(f, g, z) .
$$

Here the Jacobi identity $L(f, g, h)=0$ is used. So it suffices to show that $L(f, g, z)=0$. When $f=\bar{x}$ and $g=\bar{y}, L(\bar{x}, \bar{y}, z)=0$ is equivalent to the Leibniz identity of $\mathfrak{g}$. By the biderivation properties again,

$$
L(f \bar{x}, g, z)=f L(\bar{x}, g, z)+\bar{x} L(f, g, z) .
$$

By the assumption of induction with respect to the degree of polynomials, we have $L(\bar{x}, g, z)=L(f, g, z)=0$, which gives $L(f \bar{x}, g, z)=0$. In the same way $L(f, g \bar{x}, z)=0$ is shown.

From this lemma, we obtain the desired identity,

$$
L(f \otimes x, g \otimes y, h \otimes z)=L(f \bar{x}, g \bar{y}, h \otimes z)=0 .
$$

By a direct computation, one can check the axioms (2.4)-(2.6). Thus, $\mathcal{A}^{\text {poly }}\left(\mathfrak{g}^{*}\right)$ satisfies (A1) and (A2) in Introduction. The Leibniz algebra $\mathfrak{g}$ is identified with $1 \otimes \mathfrak{g}$ as the linear subalgebra of $\mathcal{A}^{\text {poly }}\left(\mathfrak{g}^{*}\right)$. Namely (A3) holds.

We show that $\mathcal{A}^{\text {poly }}\left(\mathfrak{g}^{*}\right)$ is the free dual-prePoisson algebra over $\mathfrak{g}$. 
Lemma 4.6. Given a dual-prePoisson algebra $P$ and given a Loday algebra homomorphism $\phi: \mathfrak{g} \rightarrow P$, there exists the unique dual-prePoisson algebra morphism $\hat{\phi}: \mathcal{A}^{\text {poly }}\left(\mathfrak{g}^{*}\right) \rightarrow P$, which is defined by

$$
\hat{\phi}:\left(\bar{x}_{1} \bar{x}_{2} \cdots \bar{x}_{n}\right) \otimes y \mapsto \phi\left(x_{1}\right) * \phi\left(x_{2}\right) * \cdots * \phi\left(x_{n}\right) * \phi(y) .
$$

Proof. The mapping $\hat{\phi}$ is well-defined because $P$ satisfies $(1 * 2) * 3=(2 * 1) * 3$ and $\{1,2\}_{P} * 3=-\{2,1\}_{P} * 3$. Here $\{,\}_{P}$ is the dual-prePoisson bracket on $P$. It is obvious that $\hat{\phi}$ preserves the perm-product. We show that $\hat{\phi}$ preserves the dual-prePoisson bracket. The defining equation (4.2) is preserved:

$$
\begin{aligned}
\hat{\phi} & \left\{\bar{x}_{1} \cdots \bar{x}_{n}, y\right\} \\
& =\hat{\phi} \sum_{i} \bar{x}_{1} \cdots \bar{x}_{i-1} \bar{x}_{i+1} \cdots \bar{x}_{n} \otimes\left[x_{i}, y\right] \\
& =\sum_{i} \phi\left(x_{1}\right) * \cdots * \phi\left(x_{i-1}\right) * \phi(x)_{i+1} * \cdots * \phi(x)_{n} *\left\{\phi\left(x_{i}\right), \phi(y)\right\}_{P} \\
& =\left\{\phi\left(x_{1}\right) * \phi\left(x_{2}\right) * \cdots * \phi\left(x_{n}\right), \phi(y)\right\}_{P} .
\end{aligned}
$$

From $\hat{\phi}(\{\bar{x}, \bar{y}\} \otimes z)=\hat{\phi}(\overline{[x, y]} \otimes z)=\phi[x, y] * \phi(z)=\{\phi(x), \phi(y)\} * \phi(x)$, for any $f, g \in S\left(\mathfrak{g}_{\text {Lie }}\right)$ we have

$$
\hat{\phi}(\{f, g\} \otimes z)=\{\hat{\phi}(f), \hat{\phi}(g)\} * \phi(z),
$$

which implies that (4.1) is also preserved.

Thus, we know that $\mathcal{A}^{\text {poly }}\left(\mathfrak{g}^{*}\right)$ satisfies (A1)-(A4). The proof of the theorem is completed.

Proof of Proposition 2.3. Since the free Poisson algebra is unital, there exists an injection,

$$
V \rightarrow 1 \otimes \mathcal{F}_{\mathcal{L} o d}(V) \rightarrow \mathcal{F}_{\mathcal{P}_{\text {oiss }}}(V) \otimes \mathcal{F}_{\mathcal{L} \text { od }}(V) .
$$

The Liezation of the free Loday algebra is the free Lie algebra, because the Liezation is universal. It is well known that $\mathcal{F}_{\mathcal{P}_{\text {oiss }}}=\mathcal{F}_{\mathcal{C O m}} \mathcal{F}_{\mathcal{L i e}}$. Hence,

$$
\mathcal{F}_{\mathcal{P}_{\text {oiss }}}(V)=\mathcal{F}_{\text {Com }}\left(\left(\mathcal{F}_{\mathcal{L}_{\text {od }}} V\right)_{\text {Lie }}\right) .
$$

Thus $\mathcal{F}_{\mathcal{P}_{\text {oiss }}}(V) \otimes \mathcal{F}_{\mathcal{L} \text { od }}(V)$ becomes a dual-prePoisson algebra. The universality is followed from the diagram:

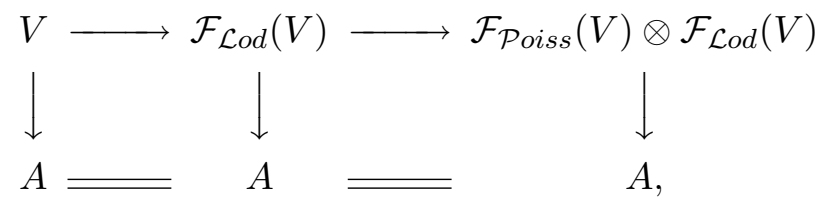

where $A$ is an arbitrary dual-prePoisson algebra. The proof of Proposition 2.3 is completed. 
We note that the projection $\mathcal{A}^{\text {poly }}\left(\mathfrak{g}^{*}\right) \rightarrow \bar{S}\left(\mathfrak{g}_{\text {Lie }}\right)$ coincides with the Poissonization of $\mathcal{A}^{\text {poly }}\left(\mathfrak{g}^{*}\right)$, that is,

$$
\left(\mathcal{A}^{\text {poly }}\left(\mathfrak{g}^{*}\right)\right)_{\text {Poiss }} \cong \bar{S}\left(\mathfrak{g}_{\text {Lie }}\right)
$$

There exists a natural morphism $\left(I_{1}, I_{0}\right)$ in $\mathcal{L} \mathcal{M}$,

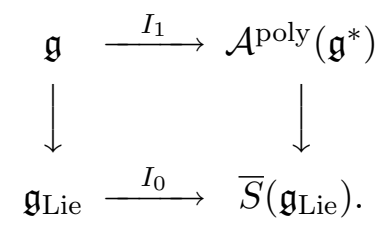

It is natural to ask whether or not $\left(I_{1}, I_{0}\right)$ is a universal arrow.

Corollary 4.7. The Poissonization $\mathcal{A}^{\text {poly }}\left(\mathfrak{g}^{*}\right) \rightarrow \bar{S}\left(\mathfrak{g}_{\text {Lie }}\right)$ is free over the Liezation, that is, this Poisson object is the "Lie-Poisson object" over the Lie object.

4.2. Smooth and graded cases. We consider a smooth case. Assume $\operatorname{dim} \mathfrak{g}<\infty$ and replace $(4.2)$ to

$$
\{f, y\}=\sum_{i} \frac{\partial f}{\partial \bar{e}_{i}} \otimes\left[e_{i}, y\right],
$$

where $f \in C^{\infty}\left(\mathfrak{g}_{\text {Lie }}\right)$ and $\left\{\bar{e}_{i}\right\}$ is a linear coordinate on $\mathfrak{g}_{\text {Lie }}^{*}$ which is identified with a linear basis of $\mathfrak{g}$. Then the definition (4.3) can be extended to $C^{\infty}\left(\mathfrak{g}_{\text {Lie }}^{*}\right) \otimes \mathfrak{g}$.

Corollary 4.8 (smooth cases). We put $\mathcal{A}^{\infty}\left(\mathfrak{g}^{*}\right):=C^{\infty}\left(\mathfrak{g}_{\text {Lie }}^{*}\right) \otimes \mathfrak{g}$. Then $\mathcal{A}^{\infty}\left(\mathfrak{g}^{*}\right)$ is a dual-prePoisson algebra.

Proof. Lemma 4.5 is shown by a direct computation without induction.

We consider the super (graded) cases. Let $V$ be a usual vector space. The parity change of $V$, which is denoted by $\Pi V$, is a superspace whose structure sheaf is the Grassmann algebra over the dual space $V^{*}$,

$$
C^{\infty}(\Pi V):=\bigwedge^{\cdot \geq 0} V^{*}
$$

If $V=\mathfrak{g}_{\text {Lie }}^{*}$ the dual space of a Lie algebra, then $C^{\infty}\left(\Pi \mathfrak{g}_{\text {Lie }}^{*}\right)$ becomes a Poisson algebra of degree $(-1,0)$. Its Poisson bracket is known as SN bracket. In the cases of Loday algebras, the superspace is not commutative algebra but perm-algebra. We introduce the Loday algebra version of SN-bracket.

Corollary 4.9 (Noncommutative SN brackets). Given a Loday algebra $\mathfrak{g}$, we define a nonstandard superspace $\Pi \mathfrak{g}^{*}$ as

$$
\mathcal{A}^{\infty}\left(\Pi \mathfrak{g}^{*}\right):=C^{\infty}\left(\Pi \mathfrak{g}_{\text {Lie }}^{*}\right) \otimes(\uparrow \mathfrak{g}) .
$$


Here $\uparrow \mathfrak{g}$ is the linear functions on $\mathfrak{g}^{*}$ with the degree +1 . Then $\mathcal{A}^{\infty}(\Pi \mathfrak{g})$ becomes a super (graded) dual-prePoisson algebra with the degree $(-1,0)$.

Definition 4.10. We call the dual-prePoisson algebras $\mathcal{A}^{\text {poly }}\left(\mathfrak{g}^{*}\right), \mathcal{A}^{\infty}\left(\mathfrak{g}^{*}\right)$ and $\mathcal{A}^{\infty}\left(\Pi \mathfrak{g}^{*}\right)$ the Loday-Poisson algebras.

Example 4.11. Let us consider the case of $\mathfrak{g}:=\operatorname{sl}(2)$. Since $s l(2)=s l(2)_{\text {Lie }}$,

$$
\mathcal{A}^{\infty}\left(\Pi s l^{*}(2)\right)=\left(\wedge^{\cdot} s l(2)\right) \otimes \uparrow \operatorname{sl}(2)
$$

In the classical case, $r=X \wedge H$ is a (triangular-) $r$-matrix which satisfies the integrability condition $\{r, r\}_{S N}=0$, where $\{,\}_{S N}$ is a classical SN bracket on $\bigwedge \mathfrak{g}$. In the noncommutative case, we obtain

$$
\begin{aligned}
\{X \otimes H, X \otimes H\}_{N S N} & =\{X \wedge H, X \otimes H\} \\
& =X \wedge\{H, X \otimes H\}-H \wedge\{X, X \otimes H\} \\
& =2 H \wedge X \otimes X,
\end{aligned}
$$

where $[H, X]=2 X$ is used.

\section{Quantization}

In this section, we study an algebraic quantization of Loday-Poisson algebra. In first, we recall the notion of associative dialgebra.

Definition 5.1 ([10]). Let $D$ be a vector space with two associative multiplications $\vdash$ and $\dashv$. When the following three axioms are satisfied, $D$ is called an associative dialgebra, or called an associative Loday algebra,

$$
\begin{aligned}
& (a \vdash b) \vdash c=(a \dashv b) \vdash c, \\
& (a \vdash b) \dashv c=a \vdash(b \dashv c), \\
& a \dashv(b \dashv c)=a \dashv(b \vdash c),
\end{aligned}
$$

where $a, b, c \in D$.

If $D$ is a dialgebra, then the commutator

$$
[a, b]_{d i}:=a \vdash b-b \dashv a
$$

is a Loday bracket. Hence, dialgebras are associative analogues of Loday algebras.

Given a Loday algebra $\mathfrak{g}$, the universal enveloping dialgebra, $U d(\mathfrak{g})$, has the form of $U d(\mathfrak{g}):=U\left(\mathfrak{g}_{\text {Lie }}\right) \otimes \mathfrak{g}$. The dialgebra multiplications on $U d(\mathfrak{g})$ are defined by

$$
\begin{aligned}
& f \otimes x \vdash g \otimes y:=f \bar{x} g \otimes y, \\
& f \otimes x \dashv 1 \otimes y:=f \bar{y} \otimes x-f \otimes[y, x], \\
& f \otimes x \dashv\left(\bar{y}_{1} \cdots \bar{y}_{n-1} \otimes y_{n}\right):=\left(\cdots\left(f \otimes x \dashv 1 \otimes y_{1}\right) \dashv \cdots\right) \dashv\left(1 \otimes y_{n}\right),
\end{aligned}
$$


where $f, g, \bar{x} . \in U\left(\mathfrak{g}_{\text {Lie }}\right)$. We have

$$
(1 \otimes x) \vdash(1 \otimes y)-(1 \otimes y) \dashv(1 \otimes x)=1 \otimes[x, y] .
$$

Since $\operatorname{gr} U\left(\mathfrak{g}_{\text {Lie }}\right) \cong S\left(\mathfrak{g}_{\text {Lie }}\right)(\mathrm{PBW}$-theorem $)$, we obtain $\operatorname{gr} U d(\mathfrak{g})=\mathcal{A}^{\text {poly }}\left(\mathfrak{g}^{*}\right)$.

Remark 5.2 ([9]). It is known that $U d(\mathfrak{g}) \rightarrow \bar{U}\left(\mathfrak{g}_{\text {Lie }}\right)$ is the universal enveloping gebra, which is an associative gebra (or associative object) in $\mathcal{L M}$, over the Lie object $\mathfrak{g} \rightarrow \mathfrak{g}_{\text {Lie }}$.

Let $S_{\star}\left(\mathfrak{g}_{\text {Lie }}\right):=\left(S\left(\mathfrak{g}_{\text {Lie }}\right)[[\hbar]], \star\right)$ be a canonical deformation quantization (Kontsevich [7]) of the Lie-Poisson algebra, where $\operatorname{dim} \mathfrak{g}<\infty$ and $\hbar$ is the formal parameter of deformation. In [7] Section 8.3.1, it was shown that $S_{\star}\left(\mathfrak{g}_{\text {Lie }}\right)$ is isomorphic to $U_{\hbar}\left(\mathfrak{g}_{\text {Lie }}\right)$ as an associative algebra, where $U_{\hbar}\left(\mathfrak{g}_{\text {Lie }}\right)$ is the universal enveloping algebra over $\mathfrak{g}_{\text {Lie }}$ with the Lie bracket $\hbar[$,$] . We$ recall the proof of this theorem. Assume $\hbar:=1$. The star-product satisfies

$$
\bar{x} \star \bar{y}=\bar{x} \bar{y}+\frac{1}{2}\{\bar{x}, \bar{y}\},
$$

on the level of generators. Since $\{\bar{x}, \bar{y}\}=[\bar{x}, \bar{y}]$, we have $\bar{x} \star \bar{y}-\bar{y} \star \bar{x}=[\bar{x}, \bar{y}]$, which gives an algebra homomorphism, $I: U\left(\mathfrak{g}_{\text {Lie }}\right) \rightarrow S_{\star}\left(\mathfrak{g}_{\text {Lie }}\right)$. This mapping is clearly surjective and preserves the top terms of polynomials. Hence, it is bijective. When $\hbar \neq 1, I$ is the map of $U_{\hbar}\left(\mathfrak{g}_{\text {Lie }}\right)$ to $S_{\star}\left(\mathfrak{g}_{\text {Lie }}\right)$, because the degree of monomial is coherent with the one of $\hbar$ :

We extend $I$ to the following isomorphism:

$$
I \otimes 1: U d_{\hbar}(\mathfrak{g}) \rightarrow \mathcal{A}_{\star}^{\text {poly }}\left(\mathfrak{g}^{*}\right),
$$

where $\mathcal{A}_{\star}^{\text {poly }}\left(\mathfrak{g}^{*}\right):=S_{\star}\left(\mathfrak{g}_{\text {Lie }}\right) \otimes \mathfrak{g}$ and where $U d_{\hbar}(\mathfrak{g})$ is the universal enveloping dialgebra over $\mathfrak{g}$ with the Loday bracket $\hbar[$,$] . Via the isomorphism, one can$ define the unique dialgebra structure on $\mathcal{A}_{\star}^{\text {poly }}\left(\mathfrak{g}^{*}\right)$. Let us denote by $\vdash_{\star}$ and $\dashv_{\star}$ the dialgebra multiplications on $\mathcal{A}_{\star}^{\text {poly }}\left(\mathfrak{g}^{*}\right)$. For example,

$$
\begin{aligned}
g \otimes & y \dashv_{\star} \bar{x}_{1} \otimes x_{2} \\
= & (I \otimes 1)\left(I^{-1}(g) \otimes y \dashv \bar{x}_{1} \otimes x_{2}\right) \\
= & (I \otimes 1)\left(\left(I^{-1}(g) \bar{x}_{1} \otimes y-I^{-1}(g) \otimes \hbar\left[x_{1}, y\right]\right) \dashv 1 \otimes x_{2}\right) \\
= & (I \otimes 1)\left(I^{-1}(g) \bar{x}_{1} \bar{x}_{2} \otimes y-I^{-1}(g) \bar{x}_{1} \otimes \hbar\left[x_{2}, y\right]-I^{-1}(g) \bar{x}_{2} \otimes \hbar\left[x_{1}, y\right]\right. \\
& \left.+I^{-1}(g) \otimes \hbar^{2}\left[x_{2},\left[x_{1}, y\right]\right]\right) \\
= & g \star \bar{x}_{1} \star \bar{x}_{2} \otimes y-\hbar g \star \bar{x}_{1} \otimes\left[x_{2}, y\right]-\hbar g \star \bar{x}_{2} \otimes\left[x_{1}, y\right]+\hbar^{2} g \otimes\left[x_{2},\left[x_{1}, y\right]\right],
\end{aligned}
$$

where $I(\bar{x})=\bar{x}$ is used. 
Proposition 5.3. For any $f \otimes x, g \otimes y \in \mathcal{A}_{\star}^{\text {poly }}\left(\mathfrak{g}^{*}\right)$,

$$
\begin{aligned}
& \lim _{h \rightarrow 0}(f \otimes x) \vdash_{\star}(g \otimes y)=(f \otimes x) *(g \otimes y), \\
& \lim _{h \rightarrow 0} \frac{1}{\hbar}[f \otimes x, g \otimes y]_{d i}=\{f \otimes x, g \otimes y\} .
\end{aligned}
$$

One concludes that $\mathcal{A}_{\star}^{\text {poly }}\left(\mathfrak{g}^{*}\right)$ is a deformation quantization of the LodayPoisson algebra $\mathcal{A}^{\text {poly }}\left(\mathfrak{g}^{*}\right)$.

Proof. By the definition of the star dialgebra products,

$$
\bar{x}_{1} \star \cdots \star \bar{x}_{n-1} \otimes x_{n} \vdash_{\star} g \otimes y=\bar{x}_{1} \star \cdots \star \bar{x}_{n} \star g \otimes y
$$

and

$$
\begin{aligned}
g & \otimes y \dashv_{\star}\left(\bar{x}_{1} \star \cdots \star \bar{x}_{n-1} \otimes x_{n}\right) \\
& =g \star \bar{x}_{1} \star \cdots \star \bar{x}_{n} \otimes y-\hbar \sum_{i \geq 1} g \star \bar{x}_{1} \star \cdots \star \bar{x}_{i}^{\vee} \cdots \star \bar{x}_{n} \otimes\left[x_{i}, y\right]+\cdots \\
& =g \star \bar{x}_{1} \star \cdots \star \bar{x}_{n} \otimes y-\hbar \sum_{i \geq 1} g \bar{x}_{1} \cdots \bar{x}_{i}^{\vee} \cdots \bar{x}_{n} \otimes\left[x_{i}, y\right]+\cdots
\end{aligned}
$$

Equation (5.1) yields the first identity of the proposition because $f$ is a polynomial with respect to the star product. The commutator is

$$
\begin{aligned}
& \lim _{\hbar \rightarrow 0} \frac{1}{\hbar}\left[\bar{x}_{1} \star \cdots \star \bar{x}_{n-1} \otimes x_{n}, g \otimes y\right]_{d i} \\
& \quad=\lim _{\hbar \rightarrow 0} \frac{1}{\hbar}\left[\bar{x}_{1} \star \cdots \star \bar{x}_{n}, g\right] \otimes y+\sum_{i \geq 1} g \bar{x}_{1} \cdots \bar{x}_{i}^{\vee} \cdots \bar{x}_{n} \otimes\left[x_{i}, y\right] \\
& \quad=\left\{\bar{x}_{1} \cdots \bar{x}_{n-1} \otimes x_{n}, g \otimes y\right\},
\end{aligned}
$$

where

$$
\lim _{\hbar \rightarrow 0} \frac{1}{\hbar}\left[\bar{x}_{1} \star \cdots \star \bar{x}_{n}, g\right]=\left\{\bar{x}_{1} \cdots \bar{x}_{n}, g\right\} .
$$

The proof of the proposition is completed.

Remark 5.4 (recall Remark 5.2). The associative object, $\mathcal{A}_{\star}^{\text {poly }}\left(\mathfrak{g}^{*}\right) \rightarrow$ $\bar{S}_{\star}\left(\mathfrak{g}_{\text {Lie }}\right)$, can seen as a quantization of the Lie-Poisson object;

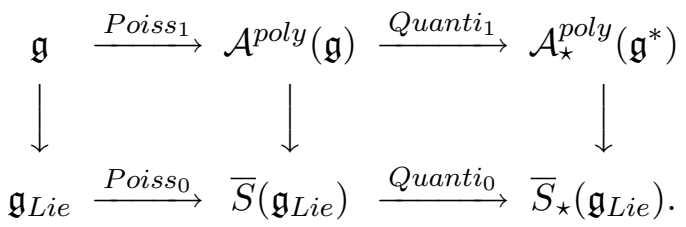

Here Poiss. is the Lie-Poisson functor and Quanti. is the quantization functor. 
Final Remark. We studied six types of algebras, i.e., three classical algebras; Lie, Poisson, Associative and their noncommutative analogues; Loday, Loday-Poisson, di-associative. We recall their operads (see $[\mathbf{4 , 5 , 1 0 - 1 2 ]}$ for operads).

Definition $5.5([\mathbf{1 3}])$. Let $\mathcal{P}$ be a binary quadratic operad and let $\mathcal{P}$ erm be the operad of perm-algebras. We call the functor

$$
\mathcal{P} \mapsto \mathcal{P} \text { erm } \otimes \mathcal{P}
$$

a derived bracket construction, on the level of operad.

It is known that the operads of the six algebras are related via the derived bracket constriction

$$
\begin{aligned}
\mathcal{L} \text { od } & =\mathcal{P} \text { erm } \otimes \mathcal{L} \text { ie }, \\
\mathcal{L} \text { odP } \text { oiss } & =\mathcal{P} \text { erm } \otimes \mathcal{P} \text { oiss }, \\
\mathcal{D} \text { ias } & =\mathcal{P} \text { erm } \otimes \mathcal{A} \text { ss },
\end{aligned}
$$

where $\mathcal{L}$ ie, $\mathcal{P}$ oiss and $\mathcal{A}$ ss are respectively operads of Lie algebras, Poisson algebras and associative algebras and $\mathcal{L}$ od, $\mathcal{L}$ odPoiss and Dias are respectively operads of Loday algebras, Loday-Poisson algebras (or dualprePoisson algebras) and associative dialgebras. Thus, we obtain an operad theoretical description for the results of the previous sections:

\begin{tabular}{|l|l|l|l|}
\hline Type & Symmetry & Classical & Quantum \\
\hline $\mathcal{P}$ erm $\otimes \mathcal{P}$ & $\mathcal{L}$ od & LodP oiss & Dias \\
\hline $\mathcal{P}$ & $\mathcal{L}$ ie & Poiss & Ass \\
\hline
\end{tabular}

\section{References}

[1] M. Aguiar, Pre-Poisson algebras. Lett. Math. Phys. 54(4) (2000), 263-277.

[2] F. Chapoton, Un endofoncteur de la categorie des operades, Lecture Notes in Mathematics, 1763. Springer-Verlag, Berlin (2001), 105-110.

[3] F. Chapoton and M. Livernet, Pre-Lie algebras and the rooted trees operad, Internat. Math. Res. Notices (8) (2001), 395-408.

[4] V. Ginzburg and M. Kapranov, Koszul duality for operads, Duke Math. J. 76(1) (1994), 203-272.

[5] V. Ginzburg and M. Kapranov, Erratum to: "Koszul duality for operands", Duke Math. J. 80(1) (1995), 293.

[6] R. Ibanez, M. de Leon, J.C. Marrero, and E. Padron, Leibniz algebroid associated with a Nambu-Poisson structure, J. Phys. A: Math. Gen. 32 (1999), 8129.

[7] M. Kontsevich, Deformation quantization of Poisson manifolds, Lett. Math. Phys. 66 (2003), 157-216.

[8] J-L. Loday and T. Pirashvili, Universal enveloping algebras of Leibniz algebras and (co)homology, Math. Ann. 296(1) (1993), 139-158.

[9] J-L. Loday and T. Pirashvili, The tensor category of linear maps and Leibniz algebras, Georg. Math. J. 5(3) (1998), 263-276. 
[10] J-L. Loday, Dialgebras, Lecture Notes in Mathematics, 1763. Springer-Verlag, Berlin, (2001), 7-66.

[11] M. Markl, S. Shnider, and J. Stasheff, Operads in algebra, topology and physics, Mathematical Surveys and Monographs, 96. American Mathematical Society, Providence, RI, (2002). x+349 pp.

[12] B. Vallette, Manin products, Koszul duality, Loday algebras and Deligne conjecture, J. Reine Angew. Math. 620 (2008), 105-164.

[13] K. Uchino, Derived bracket construction and Manin products, Lett. Math. Phys. 90 (2010), 37-53.

SHINJYUKU

TOKYO

JAPAN

E-mail address: K_Uchino@oct.rikadai.jp

Received 03/30/11, accepted 07/07/11

The author would like to thank very much the referees for useful comments and also to thank Professor Alan Weinstein for kind support. 